\title{
LA CONTRIBUCIÓN DE LA ALIMENTACIÓN PÚBLICA PARA EL DESARROLLO RURAL - REFLEXIONES A PARTIR DEL CASO COLOMBIANO
}

\author{
Nathalia Valderrama Bohórquez* \\ Sergio Schneider**
}

\begin{abstract}
Resumen
Este estudio de caso tiene como objetivo analizar cómo la alimentación pública ha sido disputada bajo diferentes concepciones, siendo un campo de confrontaciones entre diferentes actores que reconstruyen reglas y trazan trayectorias históricas discontinuas. Metodológicamente, se emplea una revisión sistemática de fuentes secundarias, así como el análisis de información primaria recolectada a través de observación participante y entrevistas semi-estructuradas a actores clave en Colombia, movilizando conceptos de la Teoría de los Campos de Acción Estratégica. La alimentación pública puede ser reposicionada como mecanismo de construcción social al fomentar las redes alternativas agroalimentarias a través de las compras locales. Sin embargo, este campo ha sido cooptado en disputas sociales y políticas por complejas dinámicas que perpetuán la apropiación de los sistemas agroalimentarios por empresas privadas e intermediarios. Por otra parte, existe una limitada capacidad del Estado por promover prácticas de producción y consumo sustentables al mismo tiempo que la normatividad fomenta modelos desconectados y asistencialistas. Este vacío estatal en algunos casos es ocupado por la acción colectiva de algunos grupos sociales con objetivos comunes. La necesidad de repensar la alimentación pública como mecanismo de desarrollo rural movilizaría y priorizaría otros significados y valores para fortalecer los intereses compartidos de bienestar.
\end{abstract}

Palabras clave: seguridad alimentaria y nutricional, inclusión social, compras locales.

\footnotetext{
*Ingeniera Agroindustrial y Master en Ciencia y Tecnología de Alimentos de la Universidad Nacional de Colombia. Estudiante de Doctorado en Desarrollo Rural de la Universidade Federal do Rio Grande do Sul (UFRGS). Porto Alegre, Rio Grande do Sul, Brasil.E-mail: nvalderramabo@unal.edu.co

* Sociólogo, Master y PhD en Sociología, Post-Doctor en la City University of London. Profesor Titular en los Programas de Postgrado en Sociología (PPGS) y Desarrollo Rural (PGDR) de la Universidade Federal do Rio Grande do Sul (UFRGS). Porto Alegre, Rio Grande do Sul, Brasil.E-mail: schneide@ufrgs.br
} 


\section{Introducción}

La sinergia entre nutrición y agricultura ha sido señalada en estudios recientes como uno de los encaminamientos para alcanzar los Objetivos del Desarrollo Sostenible (HAWKES; POPKIN, 2015; MALUF et al., 2015; PINGALLI, 2015). Según Global Panel on Agriculture and Food Systems for Nutrition (2015), la alimentación pública tiene el potencial de posicionarse como mecanismo de vinculación de procesos de producción y consumo de alimentos que pueden movilizar complementariamente flujos entre agricultura y nutrición en la política alimentaria.

Las compras públicas de alimentos pueden ser consideradas un mecanismo importante para la promoción de esta interacción a través de la utilización del poder de compra del Estado para el abastecimiento de la demanda para escuelas, universidades, hospitales y otros órganos públicos. Según, Morgan y Sonnino (2008), las compras públicas son un mecanismo de desarrollo sostenible que promueve el poder de la compra estatal. Este mecanismo se construye política y socialmente en contextos particulares como un proceso heterogéneo de imposiciones externas y preocupaciones locales. Las compras públicas están inmersas en un proceso inevitable por la actual crisis alimentaria global y su potencial radica en su capacidad de integrar tres elementos fundamentales: el desarrollo, la democracia y el ambiente. De esta forma, la compra pública tiene el potencial de equilibrar las relaciones de poder dentro del sistema agroalimentario para posicionarse como mecanismo de desarrollo sostenible con múltiples efectos a favor de la inclusión social, económica y política de comunidades excluidas.

La alimentación pública se interrelaciona con los debates actuales de la alimentación, los cuales reiteran la crisis del paradigma productivista a nivel global y demuestran sus limitaciones en los campos del ambiente y la salud pública. Al mismo tiempo se consolidan las bases para la emergencia de nuevos paradigmas, los cuales disputan entre sí espacios y mecanismos de argumentos, normatividades y reconocimiento (LANG; HEASMAN, 2004).

Pero hay otras dimensiones que pueden ser exploradas, ya que las compras de alimentos por el Estado pueden conducir a dinámicas en las cuales la producción de alimentos garantice la diversificación de las dietas y el acceso a renta que fomenta la autonomía y el empoderamiento de actores sociales con impacto directo en la condición nutricional de las familias (HERFORTH; HARRIS, 2014). Según World Food Programme (WFP, 2012, 2013), algunos resultados de esta sinergia se relacionan con la superación de los ciclos intergeneracionales de pobreza, el empoderamiento y la organización, la protección social y el desarrollo humano y la permanencia y reproducción en el rural. 
Sin embargo, estudios recientes señalizan que los programas de alimentación encaran desafíos para el cumplimiento de sus objetivos debido a dificultades asociadas con la baja capacidad de integrar diferentes elementos dentro de los programas (FAO, 2013; GALAA; SAAKA, 2011; GELLI; SUWA, 2014; GELLI; ESPEJO, 2013). La limitada posibilidad de hacer efectivo este vínculo a partir de la compra directa de la producción campesina de alimentos en los programas de alimentación públicos se debe a que existe un mercado apropiado por grupos sociales más capacitados que controlan y acaparan los medios de producción y a través de alianzas con otros grupos imponen modelos excluyentes que reproducen la condición de desventaja y vulnerabilidad de las comunidades beneficiarias, limitando la capacidad de agencia de la comunidad y reduciendo la alimentación pública a una concepción meramente asistencialista. Estos modelos excluyentes son incapaces de promover el desarrollo rural y comunitario.

En este artículo intentaremos analizar cómo la alimentación pública ha sido disputada bajo diferentes concepciones, siendo un campo de confrontaciones entre diferentes actores que reconstruyen reglas y trazan trayectorias históricas discontinuas. En primer lugar, se tejen conceptualmente las relaciones entre la alimentación pública y el desarrollo rural en base a los estudios publicados sobre el tema. Seguidamente se presenta el estudio de caso de la alimentación pública en Colombia enfocando específicamente en la adquisición de alimentos y analizando estos programas como campos en disputa. Finalmente, se discute las lecciones aprendidas para contribuir al debate internacional de la alimentación pública como mecanismo de desarrollo rural.

\section{Los programas públicos de alimentación como mecanismo de desarrollo rural: quebrando el paradigma del asistencialismo}

Las limitaciones de acceso a alimentos suficientes, inocuos y nutritivos comprometen la salud y el desarrollo físico y cognitivo de las personas. Sin embargo, el hambre frecuentemente hecha invisible, naturalizada y negada, trasciende del campo biológico-clínico al ser una manifestación de las desigualdades producto de procesos históricos, sociales, políticos y económicos de exclusión y de falta de proyectos estructurales transformadores (FREITAS, 2003). A largo plazo, el hambre transmite, perpetúa e intensifica los ciclos y las dinámicas de pobreza y de vulnerabilidad económica y social, por lo que uno de los principales desafíos de las zonas rurales y urbanas continúan siendo la superación del hambre y la reducción de la pobreza.

La alimentación pública emerge para brindar mejores condiciones de vida y ejercer una influencia positiva en la alimentación al permitir el acceso 
a alimentos más saludables, de mejor calidad y adecuados culturalmente, minimizando también la exclusión socioeconómica, así como creando oportunidades para el desarrollo económico de los productores de alimentos en pequeña escala, orgánicos y provenientes del comercio justo (MORGAN; SONNINO,2008). De esta forma, el plato público trasciende de la cantidad de ración entregada a la esfera pública de decisiones políticas, siendo que la alimentación pública es promotora de reconocimiento, de desarrollo social y de seguridad alimentaria y nutricional (MORGAN; MORLEY, 2014).

Adicionalmente, relatos de experiencias y estudios a nivel internacional han demostrado que la vinculación de la agricultura y la nutrición para el abastecimiento de los programas de alimentación pública conllevan a resultados positivos como el aumento en la compra de hortalizas y frutas (AMORIM; ROSSO; BANDONI, 2016; BARONE et al., 2016; SOARES et al., 2017), el incremento de la capacidad de cobertura y sustentabilidad de los programas (FOEKEN; OWUOR; MWANGI, 2010), así como procesos y espacios de decisión que fomentan la seguridad alimentaria y nutricional, el desarrollo rural sustentable y la producción agroecológica (CONSTANTY; ZONIN, 2016; ANÓNIMO, 2010; OLIVEIRA; BATALHA; PETTAN, 2017; UDUKU, 2011; BORISH; KING; DEWEY, 2017). Así mismo, la formulación e implementación de políticas públicas que integran circuitos cortos en sistemas alimentarios descentralizados en el caso brasileiro han sido capaces de promover modelos de producción sustentables de base agroecológica, el acceso a dietas más saludables y diversificadas, la reducción en las pérdidas y desperdicios y en los costos energéticos, de transporte y logísticos, a pesar de que el sector del agronegocio continúe reproduciendo dietas no sustentables ni saludables (MALUF et al., 2015).

Las concepciones basadas en el aumento de la eficiencia en el gasto púbico, la contratación pública con empresas privadas y la padronización de las operaciones logísticas ha influenciado negativamente conduciendo a la elección de los menores precios, dietas con alto contenido calórico y la pérdida de la diversidad alimentaria (MORGAN; MORLEY, 2014). Adicionalmente, varios estudios apuntan que los fenómenos de exclusión de los agricultores persisten debido a una ausencia o un diseño ineficiente de programas y políticas públicas diferenciadas, un complejo aparato burocrático que limita la producción y compra, así como la incapacidad de garantizar dinámicas y mecanismos de gestión que respalden iniciativas inclusivas (RUSSELL et al., 2008; BEESLEY; BALLARD, 2013; SOUZA-ESQUERDO; BERGAMASCO, 2014; MARQUES; BARATA; ALVES, 2016; SOARES et al., 2015; SULEMANA; NGAH; MAJID, 2013; VILLAR et al., 2013). 
Las discusiones sobre alimentación pública se posicionan en campos donde se disputan recursos, significados y cuadros normativos entre los distintos atores. Estos campos son socialmente construidos, también de forma conflictiva, y están siendo influenciados constantemente por múltiplos factores internos y externos. Estos factores inminentemente se encuentran sometidos a procesos de inestabilidad y a la emergencia de nuevas estrategias de contención (FLIGSTEIN, 2002). El presente artículo moviliza conceptos de la Teoría de los Campos de Acción Estratégica reconociendo la emergencia, estabilidad y transformación política, así como también los mecanismos de reconocimiento, de movilización significados y de poder entre los actores involucrados para el caso del análisis de las trasformaciones de la alimentación pública en Colombia ${ }^{1}$.

\section{La alimentación pública como campo en disputa en Colombia}

En Colombia, los programas de alimentación pública fueron mecanismo de desarrollo rural años atrás. A pesar de que estos programas no estaban concebidos exclusivamente para este fin, la acción comunitaria de las familias rurales y la gestión estatal del Instituto Colombiano de Bienestar Familiar a partir de la década del 80 fueron los promotores de este mecanismo.

Fue solamente hasta la década del 80 , que se crearon las modalidades de gestión comunitaria a través de procesos de concertación y mediación del ICBF (CASTILLO MATAMOROS, 2009; URIBE, 1988). Esto se fundamentó en la necesidad de aumentar la cobertura y la efectividad de los programas usando un presupuesto público limitado y asegurando una cobertura a bajo costo. A través de estas modalidades comunitarias, las asociaciones de padres de familia, entidades sin ánimo de lucro y Juntas de Acción Comunal administraban y gestionaban los programas de alimentación.

Rescatando las declaraciones de algunos de los interlocutores ${ }^{2}$ se evidencia que la organización comunitaria conllevaba a resultados positivos en relación con la calidad y eficiencia del servicio prestado.

"En ese entonces cada institución organizaba el comité de padres de familia, eran ellos (Juntas de Padres de Familia) quienes recibian esta plata y ellos eran los que distribuían" [...]. "El servicio era mucho mejor. Siempre y cuando las Juntas fueran muy honradas, muy prestantes y disponibles para trabajar". (Interlocutor 1, 26 de febrero de 2018).

"[...] se facilitaba la compra de productos en la misma vereda a menor costo, con mayor calidad, sin deterioro por desplazamiento y en cantidades generosas porque los campesinos no escatiman si pueden aportar un poco más[...]" (:ंPor qué cree que la propuesta funcionó y a la gente le gustó?) "Primero porque no tenían que cargar los productos, los tenían en la misma
1 Se comprende como Campo de Acción Estratégica al espacio social donde los actores posicionan, negocian e intercambian significados y entendimientos para asegurar su reproducción social, sobrevivencia y posición en un conflicto abierto y en una constantemente estabilidad/inestabilidad del campo (FLIGSTEIN; MCDOUG, 2012).

240 entrevistas semiestructuradas fueron realizadas durante un trabajo de campo durante tres meses en 3 municipios de Colombia: Samaniego (Nariño), Florencia (Caquetá) y Granada (Antioquia). Adicionalmente, fueron aplicadas tres técnicas más de recolección de datos: análisis documental, observación participante y diario de campo. Los principales grupos de actores clave fueron gestores de los programas (públicos y privados) profesores, y campesinos. Los nombres de los participantes se mantienen en anonimato por solicitud de los interlocutores. 
vereda. Segundo porque les llegaban frescos y sanos porque los conseguian abi mismo, no era una mora estripada, ni una guayaba que llegara en malas condiciones. Tercero, porque ellos hacian un muy buen uso de los recursos. Siempre a fin de año a todas las Juntas (de Acción Comunal) les quedaba plata" [...] "La optimización del presupuesto fue una gran fortaleza de la operación del programa con el este esquema, pues cuando se hacía con un proveedor externo casi nunca alcanzaba el recurso asignado para cubrir todo el ciclo escolar, dejando periodos de tiempo escolar sin el servicio de restaurante." (Interlocutor 2, 12 de abril de 2018).

"[...] Si nos quedaban excedentes, con esos excedentes al otro año iniciábamos el restaurante [...]". "Entonces fue una experiencia muy buena porque nos permitió a nosotros darle al estudiante y a los niños lo que realmente estaban necesitando, mientras que en este momento nos toca acomodarles con lo que nos llega". (Interlocutor 3, 8 de abril de 2018).

Estos procesos comunitarios fueron paulatinamente sustituidos por modelos padronizados de operación de los programas en conjunto con otros mecanismos excluyentes que incluyen certificaciones de calidad, así como normatividades sanitarias y contractuales. Esta situación se ha vuelto ineficiente desde la última década y más crítica en los últimos años. Estos fenómenos se han ido presentando desde los años 90, cuando la intervención del Estado fue reducida y sus funciones de prestación de servicios públicos y sociales fueron delegadas al sector privado, lo que conllevó a la dificultad de controlar la calidad de los servicios y propició la formación de monopolios (OCAMPO, 1992). Estos cambios ocurrieron dentro de un nuevo modelo de desarrollo económico marcado por la ideología neoliberal que redujo el papel del Estado, en un contexto de apertura económica y de política de racionalización de la inversión pública. Como resultado hubo un desmonte de la institucionalidad pública, una mercantilización de los servicios públicos y sociales, y consecuentemente la paralización o focalización de varios programas (QUINTERO, 2010). Así mismo, la política fiscal sustentada en valores de eficiencia económica y enmarcada en una lógica de reestructuración administrativa continuó propiciando desequilibrios regionales y condicionó los proyectos de desarrollo local a los patrones impuestos por los planes de desarrollo nacional (FAJARDO, 1991; INSTITUTO DE ESTUDIOS REGIONALES, 2000; MUÑOZ; MARTÍNEZ; CARREÑO, 1993).

Consecuentemente, las entidades públicas han diseñado complicados pliegos de contratación que han subordinado los valores asociados a la calidad de los alimentos, al reconocimiento de los hábitos alimentarios de la población y a la gestión comunitaria de los recursos públicos. Algunas declaraciones de los interlocutores demuestran que estos marcos normativos han transformado las dinámicas de la compra de alimentos y han excluido la participación de la comunidad en los procesos con serios efectos sobre la calidad de la alimentación. 
"El frijol lo compran acá por bultos al campesino y cualquier empresa lo empaca y lo vuelve a traer acá”. (Interlocutor 4, 9 de mayo de 2018).

"Por lo general (antes) los alimentos se compraban en el pueblo, pero también habia alimentos que se podian comprar en el campo, la panela, el buevo, el revuelto, el plátano, la yuca, todas esas cositas asi las podíamos comprar en el campo. Ahora es muy difícil... porque requieren que el registro Invima, que esto, que lo otro, que las gallinas estén vacunadas. Una cantidad de cosas que uno por ser pequeño productor no puede garantizar. Entonces es muy triste también porque la administración (municipal o departamental) tiene la oportunidad de comprarle al campesino, pero desde la misma gobernabilidad lo impide [...]”. (Interlocutor 3, 8 de abril de 2018).

"Entre más dias más estricto. Cuando nosotros iniciamos, nosotros podiamos comprar huevos acá, incluso cuando iniciamos el programa con madres comunitarias nosotros podíamos dar leche de vaca de la botella, ya no" [...] "Acá hay varias empresas que producen productos de aseo para limpiar como el ambientador para el piso, los traperos, pero no se pueden comprar porque no tienen Invima, no tienen los permisos que exige la norma”. (Interlocutor 5, 19 de febrero de 2018).

"[...] Con los comités ahora los padres de familia casi no vienen y ellos deberian venir, mirar la minuta, verificar que lo que diga la minuta es lo que se les está dando a los niños, verificar que no solamente sea lo de la minuta sino también lo de las cantidades que dice la minuta. Digamos que el padre de familia, con tal de que el niño reciba algo cualquier cosa que sea le parece bien”. (Interlocutor 6, 12 de febrero de 2018).

De esta forma, la priorización de ciertos cuadros normativos restrictivos ha sido la forma de legitimar la ausencia estatal y estabilizar el campo ${ }^{3}$. Sumado a esto, la anarquía del sistema agroalimentario debido a problemas macro-estructurales también contribuyen a la reproducción social del sector privado y limita la reproducción social y el bienestar de las comunidades rurales (PATIÑO et al., 1988). Así, los procesos de creación, interpretación y soporte de significados, identidades, intereses y mundos compartidos para algunos grupos de actores estratégicos (intermediarios y operadores privados) se consolidan, en cuanto para otros grupos el conjunto de factores que moldean el campo de disputa no resulta conjugarse y alinearse para permitir su reproducción (FLIGSTEIN; MCDOUG, 2012, p. 51). Lo anterior, ha colocado a los operadores privados e intermediarios como grupos dominantes ya que ocupan un lugar privilegiado y movilizan otro tipo de recursos dentro de estos campos disputados, como lo indica las siguientes declaraciones:

"Ya en el transcurso del tiempo, yo he notado que el restaurante ha tenido unas condiciones más precarias. Precarias en el sentido de que los recursos son muy limitados, es más, ya ni siquiera ofrecen la minuta para un almuerzo, sino para un desayuno [...] (¿.Si fuera ese solamente
3 Según Fligstein (2002), las normas son mecanismos que definen las relaciones de competición y cooperación, determinan las negociaciones y acuerdos, y consecuentemente delimitan el rol de los actores dentro de los campos. 
el alimento que reciben sería suficiente para alimentar a todos los niños?). "No, no, no, no es suficiente. Ya eso lo hemos mirado, porque ellos envian es por gramos, ellos controlan eso es por gramaje y ya los muchachos de décimo que son jóvenes comen bastante. Si le va a servir 5 cucharadas de arroz con eso no va a quedar satisfecho el estudiante. Si le va a servir solamente un cucharón de sopa, no. A los grandes les dan 2 cucharones o cucharón y medio". (Interlocutor 7, 7 de abril de 2018).

"La cantidad de alimentos es relativamente baja para los estudiantes y no es acorde con las costumbres que se tienen acá"[...]. "En realidad, las comunidades educativas no tenemos mucha injerencia en cuanto a los alimentos que nos entregan y de acuerdo a la clase de recursos que se manejen. Nos dan simplemente informes generales". (Interlocutor 8, 27 de febrero de 2018).

"Es pura politiqueria, le entregan (el contrato) a alguien que ha invertido tanta plata en la campaña y ese es. Esa es la realidad. Es triste que esos proyectos que deberían beneficiar a toda la comunidad, a todos los estudiantes" [...] "El problema es que se queda en todo, en el transportador, el que empaca. En toda esa cadenita ahi, queda más de la mitad". (Interlocutor 9, 26 de febrero de 2018).

[El Gobierno] "[...] como que todavía no tiene mucha confianza en los pequeños productores para atender un programa de estos, más lo hace es con grandes empresarios que ellos poco tienen en cuenta el pequeño productor" [...] "Yo no sé porque no tienen en cuenta esto, si esto ayuda mucho al crecimiento de la región. Se lo dan a uno, dos o tres grandes comerciantes que ellos si tienen la capacidad financiera para atender un Programa de Alimentación Escolar, pero no pueden pensar en un pequeño productor." (Interlocutor 10, 8 de febrero de 2018).

A pesar de los contextos de exclusión que encaran las comunidades rurales, existen espacios de disputa en los cuales múltiples actores podrían reconocer y posicionar la alimentación pública como mecanismo para el desarrollo rural. Se identifica, en primer lugar, que la descentralización en la toma de decisiones sobre los programas públicos en alimentación puede crear espacios de diálogo y articulación entre diferentes actores a nivel municipal. Esto conlleva a procesos de negociación efectivos para la adquisición de géneros alimenticios producidos por asociaciones campesinas y la gestión comunitaria de los programas, al mismo tiempo en que se priorizan los proyectos construidos colectivamente en consonancia con las dinámicas de desarrollo local.

A pesar de que en la mayoría de los casos no se efectúe la compra directa dentro de los programas, emergen continuamente estrategias que son reflejo de la acción colectiva de las comunidades locales, las cuales muchas veces son invisibilizadas. La crítica situación de la alimentación pública en Colombia es promotora de acciones comunitarias que suplen las deficiencias de los programas, las cuales la institucionalidad no es capaz de 
resolver. Según García, Restrepo y Triana (2007), la acción colectiva a través de la participación activa y los espacios de concertación comunitaria han conducido a procesos de unificación de voluntad y de acción, a diferencia de las intervenciones y acciones institucionales de entidades estatales que han sido incapaces de generar una apropiación y compromiso por parte de la comunidad.

En el estudio de caso, se identificaron que las comunidades emplean diversas estrategias en pro de la alimentación de la infancia y la adolescencia, con el objetivo de llenar el plato vacío de estas poblaciones. En primer lugar, existen contribuciones en dinero o alimentos que la comunidad (padres de familia, profesores o madres comunitarias) aporta de forma voluntaria con previo acuerdo mutuo. Así mismo, se organizan actividades sociales y culturales con el fin de generar fondos que contribuyan a completar las raciones para brindar la alimentación que se requiere. De forma casi unánime, la comunidad considera que los alimentos que se reciben dentro de los programas no son suficientes para asegurar un consumo adecuado, por lo que es necesario acomodarles o mejorarles la minuta. Finalmente, se identificaron algunos casos de trueques de alimentos de forma estratégica en los mercados locales, así como la complementación con las hortalizas y las legumbres que provienen de huertas escolares y comunitarias.

Actualmente, pese a los esfuerzos de introducir las compras locales dentro de los lineamientos de estos programas y propiciar la consecución de una paz estable y duradera después de la firma del acuerdo con las FARC-EP, las normatividades han sido ineficaces para la construcción social de estos mercados institucionales. A nivel nacional, las instituciones que conforman la Comisión Intersectorial de Seguridad Alimentaria y Nutricional de Colombia - CISAN, no han posicionado el tema de compras públicas en la agenda por la dificultad de articularlo como una responsabilidad intersectorial y por falta de la representación que sea capaz de liderar y encabezar esta estrategia. Por otra parte, los esfuerzos de las organizaciones internacionales han sido insuficientes y los avances reducidos debido al limitado espacio de influencia política dentro de un campo de discusiones infértiles y de planes de desarrollo con objetivos contradictorios. Finalmente, se reconoce la necesidad de permitir la representación política de los campesinos y de la sociedad civil en espacios públicos y de veeduría que permitan el diseño y la implementación de políticas, programas y planes más efectivos e inclusivos.

Lecciones aprendidas: la alimentación pública como proceso en construcción

Analizando el caso de estudio, en primer lugar, se evidencia que las reglas de transacción y las estructuras de gobernanza que definen la forma 
de gestionar los programas colocan a las asociaciones de agricultores y a la comunidad escolar como actores desafiadores, siendo que ocupan espacios menos privilegiados y su influencia sobre el campo es reducida y condicionada al esquema dominante (FLIGSTEIN; MCDOUG, 2012). Por otra parte, los operadores privados e intermediarios (actores beneficiarios) dentro de su lógica de búsqueda por lucro económico reducen sus operaciones a transacciones comerciales fuera de los municipios, subordinan la capacidad de agencia y toma de decisión de las comunidades locales y propician la desintegración de los sistemas agroalimentarios y la apropiación de valor por un número reducido de actores. Así mismo, el Estado desde una posición sumisa continúa relegando su papel de garantizar el derecho a una alimentación adecuada para los grupos que acceden a estos programas y los reduce a procesos estandarizados que solamente velan por el cumplimiento de los contratos o las cantidades de alimento establecidas, sin promover hábitos de consumo sustentables, la inserción de las asociaciones de productores como abastecedores directos ni procesos de desarrollo local y comunitario

Las dinámicas que reposicionan la alimentación pública no son continuas ni lineales, estas conducen a factores que conllevan a avances y retrocesos en las trayectorias que reconfiguran sus significados y cuadros normativos.

A pesar que en Colombia y en el mundo, los programas públicos de alimentación y de asistencia buscan mejorar la condición nutricional de grupos focalizados con impactos transversales en otros componentes, se identifican limitaciones. La concepción que reduce los programas de alimentación a mecanismos de asistencia social no ha permitido la apertura y potencialización de las discusiones. Sin embargo, múltiples experiencias y estudios señalan las oportunidades y beneficios múltiples que estos programas tienen para reducir los vacíos y conectar los vínculos perdidos dentro del sistema alimentario de abastecimiento, en especial en las zonas rurales donde los productores y los consumidores en un grande porcentaje tejen relaciones vecinales y de parentesco.

En la medida en que se permita el acceso de los productores campesinos a mercados institucionales estables dentro de los mismos municipios, la agricultura se posicionaría como una fuente rentable de generación de ingresos familiares y la alimentación pública tomaría otro significado, que trasciende las fronteras de generación de lucro.

Así mismo, la creación de redes alternativas agroalimentarias y la simplificación de las operaciones dentro del sistema de abastecimiento producto de la venta y entrega directa de algunos alimentos frescos en las zonas rurales tuvieron efectos positivos sobre la eficiencia de los procesos. De esta forma, la reducción de las distancias, del tiempo de transporte y 
de los actores intermediarios condujo a resultados positivos asociados a la conservación de la frescura de los alimentos, a la reducción del precio de los productos y del gasto de energía, tiempo y dinero durante el transporte. Por otra parte, pueden emerger relaciones sociales de cercanía entre productores y consumidores beneficiando mutuamente al conjunto de actores.

Las compras directas a las asociaciones de productores pueden fomentar la activación de las economías campesinas, generar una redistribución de la renta entre los agricultores y aumentar la capacidad de reinvertir los recursos en otras actividades familiares agropecuarias y no agropecuarias. Por lo anterior, la alimentación pública debería posicionarse en Colombia como un mecanismo efectivo de generación de empleo, activación de las economías locales, retorno de las personas desplazadas y víctimas del conflicto armado y como una alternativa estable para la reproducción económica y social de las poblaciones rurales.

\section{Consideraciones finales}

Los programas públicos de alimentación han sido transformados conforme diferentes contextos históricos nacionales e internacionales, así como han sido influenciados por negociaciones y disputas de concepciones, reglas e intereses que influenciaron en la definición del rol que los múltiples actores desempeñan, de los significados de la alimentación y de la forma de gestión de los programas.

En un esfuerzo por rescatar sus trayectorias históricas y analizar cómo los agricultores campesinos se han vinculado a los programas a través de las compras directas, se resalta que estos procesos sociales han sido dinámicos y discontinuos.

Los cimientos que soportaban la gestión de la alimentación pública en sus primeros años, en los años 30, desconocían la diversidad alimentaria de la región. En los años ochenta en Colombia, la alimentación ganó importancia transversal en la agenda pública debido al posicionamiento de políticas para la atención a la infancia y la adolescencia que estaban siendo forjadas a la par de las bases democráticas del país y de procesos de gestión comunitaria y participación política que antecedieron los años noventa. Sin embargo, la crisis fiscal y las políticas de racionalización de la inversión pública de los años noventa dentro de un contexto de apertura económica y un desmonte de la institucionalidad pública, limitaron la capacidad y autonomía de las organizaciones de base de gestionar recursos estatales e incentivaron la transferencia de responsabilidades del sector público a empresas privadas. Estas últimas continuamente se han apropiado de espacios de agencia social, han reconfigurado la gestión de los programas de alimentación pública y del sistema de abastecimiento agroalimentario, 
y han moldeado los significados de la alimentación, reduciéndola a ser un mercado generador de lucro.

Algunos cuadros normativos propician directa e indirectamente el reconocimiento, la posición privilegiada y la reproducción de empresas privadas que mantienen las relaciones de dominación de los monopolios de empresas operadoras de servicios de alimentación y de los intermediarios en los sistemas agroalimentarios, así como perpetúan la desconexión entre producción y consumo y limitan las posibilidades de implementar mecanismos eficientes para reducir las inequidades económicas y sociales de las poblaciones. Por otra parte, estos cuadros normativos subordinan la participación de las asociaciones de agricultores y de la comunidad escolar, los cuales ocupan la posición menos privilegiada, así como tienen una influencia reducida en los campos de acción.

La vinculación directa de la producción de alimentos a los programas públicos en alimentación acarrea múltiples desafíos y oportunidades cuando se analiza la alimentación pública como mecanismo de desarrollo rural. En el caso colombiano, la agricultura tiene un enorme potencial para posicionarse como mecanismo de desarrollo al ser una fuente rentable de generación de ingresos familiares. Al vincular la agricultura a la alimentación pública se fomenta un efectivo mecanismo de comercialización al eliminar la intermediación que mantiene a los programas como sistemas de abastecimiento poco eficientes, desconectados y donde operan múltiples mecanismos de dominación y apropiación de valor. Así mismo, al permitir espacios de participación y toma de decisión comunitaria, la alimentación cobraría importancia como mecanismo de construcción social con el cual se posicionan nuevos significados y valores conectando recíprocamente los campos de producción y el consumo a través de redes alternativas agroalimentarias, las cuales son movilizadas y moldeados por intereses compartidos de bienestar.

\section{Agradecimientos}

Los autores agradecen al Conselho Nacional de Desenvolvimento Científico e Tecnológico (CNPq) y al Ministério da Ciência, Tecnologia, Inovações e Comunicações al ser el estudio parte del proyecto de investigación apoyado por la convocatoria CNPq/MCTIC nº 016/2016 Seguridad Alimentaria y Nutricional en el ámbito de la Unasur.

\section{Referencias}

AMORIM, Ana Laura Benevenuto de; ROSSO, Veridiana Vera de; BANDONI, Daniel Henrique. Acquisition of family farm foods for school meals: Analysis of public procurements within rural family farming published by the cities of São 
Paulo state. Revista de Nutrição, v. 29, n. 2, p. 297-306, 2016. Disponible en: <http://dx.doi.org/10.1590/1678-98652016000200012>. Acceso en: 12 feb. 2017.

ANÓNIMO. The Gardens for Life project. Appropriate Technology, v. 37, n. 1, p. 63, 2010.

BARONE, Bruna; NOGUEIRA, Rosana Maria; BARROS, Thiara Teixeira de; RIBEIRO, Tatiane Godoy; QUEIROZ, Kátia Regina Leoni Silva Lima de; BEHENZ, Jorge Herman; RODRIGUES, Nilo; SABBIÃO, Sérgio. Oferta de frutas e hortaliças da agricultura familiar na alimentação escolar. Vigilância Sanitária em Debate: Sociedade, v. 4, n. 3, p. 86-95, 2016. Disponible en: <http:// dx.doi.org/10.22239/2317-269x.00758>. Acceso en: 3 mar. 2017.

BEESLEY, Alan; BALLARD, Richard. Cookie cutter cooperatives in the KwaZulu-Natal school nutrition programme. Development southern Africa, Sandton, v. 30, n. 2, p. 250-261, 2013. Disponible en: <https://doi.org/10.1080/03 76835X.2013.801195>. Acceso en: 23 feb. 2017.

BORISH, David; KING, Nia; DEWEY, Cate. Enhanced community capital from primary school feeding and agroforestry program in Kenya. International Journal of Educational Development, v. 52, p. 10-18, 2017. Disponible en: <https://doi. org/10.1016/j.ijedudev.2016.10.005 >. Acceso en: 6 dic. 2017.

CASTillo MATAMOROS, Sara Eloísa del. La Génesis del Programa de Hogares Comunitarios del Instituto Colombiano de Bienestar Familiar. Tesis (Doctorado en Ciencias Sociales) - Niñez y Juventud, Universidad de Manizales, Centro de Estudios Avanzados en Niñez y Juventud, Manizales, Colombia, 2009. Disponible en: <http://biblioteca.clacso.edu.ar/Colombia/alianza-cindeumz/20130225051642/tcastillosara.pdf>. Acceso en: 6 mar. 2017.

CONSTANTY, Hadrien François Pierre-Henri; ZONIN, Wilson João. Programa Nacional de Alimentação Escolar (PNAE) e sustentabilidade: o caso do município de Marechal Cândido Rondon. Desenvolvimento e Meio Ambiente, v. 36, p. 371392, 2016. Disponible en: <http://dx.doi.org/10.5380/dma.v36i0.39688>. Acceso en: 10 mar. 2017.

FAJARDO, Darío. Experiencias en planificación descentralizada del desarrollo rural con participación campesina. In: La descentralización en Colombia. Estudios y propuestas. compilado por Jean-Michel Blanquer y Darío Fajardo. Bogotá: Universidad Nacional de Colombia, Institut français d'études andines (IFEA), 1991. p. 83-103. Disponible en: <http://www.bdigital.unal.edu.co/1422/5/04CAPI03. pdf>. Acceso en: 10 mar. 2017.

FAO. Alimentación escolar y las posibilidades de compra directa de la agricultura familiar. Estudio nacional de Colombia, 2013. Disponible en: $<$ http:// www.fao.org/docrep/field/009/as513s/as513s.pdf>. Acceso en: 6 mar. 2017.

FLIGSTEIN, Neil. The architecture of markets. An Economic sociology of twenty-first-century capitalist societies. Reino Unido: Princeton University Press, 2002.

; MCADAM, Doug. The theory of fields. New York: Oxford University Press, 2002.

FOEKEN, Dick, OWUOR, Samuel O.; MWANGI, Alice M. Urban School Farming to Improve School Feeding: The Case of Nakuru Town, Kenya. Children 
Youth and Environments, v. 20, n. 1, p. 276-300, 2010. Disponible en: <http:// www.jstor.org/stable/10.7721/chilyoutenvi.20.1.0276?seq=1\#page_scan_tab_ contents>. Acceso en: 3 mar. 2017.

FREITAS, Maria do Carmo de. Agonia da Fome. Rio de Janeiro: Editora FIOCRUZ; Salvador: EDUFBA, 2003. Disponible en: <https://static.scielo.org/ scielobooks/r9y7f/pdf/freitas-9788575412497.pdf>. Acceso en: 12 feb. 2017.

GALAA, Sylevester; SAAKA, Mahama. Running an effective and sustainable School Feeding Programme: Key factors to consider. Journal of Social Development in Africa, v. 26, n. 2, p. 39-65, 2001. Disponible en: <https://www. researchgate.net/profile/Mahama_Saaka/publication/234126672_Running_an_ effective_and_sustainable_school_feeding_programme_Key_factors_to_consider/ links/0912f50f689d88e121000000.pdf>. Acceso en: 1 mar. 2017.

GARCÍA, Geovana Benilda; RESTREPO, Gloria Rocío Restrepo; TRIANA, Luz Marina. Estudio de caso: hogares de La Casona y El Limonar Dosquebradas. Tesis (Grado en Licenciatura en Etnoeducación y Desarrollo Comunitario) UniversidadTecnológica de Pereira,Pereira,Colombia,2007.Disponible en: <http:// repositorio.utp.edu.co/dspace/bitstream/handle/11059/765/3620425G216po. pdf? sequence=1>. Acceso en: 20 mar. 2017.

GELLI, Aulo; ESPEJO, Francisco. School feeding, moving from practice to policy: reflections on building sustainable monitoring and evaluation systems. Public Health Nutrition, v. 16, n. 6, p. 995-999, 2013. Disponible en: <https:// doi.org/10.1017/S1368980012003989 >. Acceso en: 10 mar. 2017.

.; SUWA, Yuko. Investing in innovation: Trade-offs in the costs and costefficiency of school feeding using community-based kitchens in Bangladesh. Food and Nutrition Bulletin, v. 35, n. 3, p. 327-337, 2014. Disponible en: <https://doi. org/10.1177/156482651403500305>. Acceso en: 10 mar. 2017.

GLOBAL PANEL ON AGRICULTURE AND FOOD SYSTEMS FOR NUTRITION. Healthy meals in schools: policy innovations linking agriculture, food systems and nutrition. 2015. Disponible en: <http://www.glopan.org/sites/ default/files/pictures/PolicyBrief_healthyMeals.pdf>. Acceso en: 6 dic. 2017.

HAWKES, Corinna; POPKIN, Barry M. Can the sustainable development goals reduce the burden of nutrition-related non-communicable diseases without truly addressing major food system reforms? BMC Medicine, v. 13, p. 143, 2015. Disponible en: <https://doi.org/10.1186/s12916-015-0383-7>. Acceso en: 8 mar. 2017.

HERFORTH, Anna; HARRIS, Jody. Understanding and applying primary pathways and principles. Brief \#1. Improving nutrition through agriculture technical brief series. Arlington, VA: USAID/Strengthening Partnerships, Results, and Innovations in Nutrition Globally (SPRING) Project, 2014. Disponible en: $<$ https://www.spring-nutrition.org/sites/default/files/publications/briefs/spring understandingpathways_brief_1.pdf>. Acceso en: 15 feb. 2017.

INSTITUTO DE ESTUDIOS REGIONALES. Bajo Cauca. Desarrollo regional: una tarea en común universidad-región.Medellín:INER,2000.Disponible en: <http:/www.udea.edu.co/wps/wcm/connect/udea/c3957be6-8230-4d1f9e41-845154328ce5/caracterizacion-bajo-cauca.pdf?MOD=AJPERES $>$. Acceso en: 10 jun. 2017. 
LANG; Tim; HEASMAN, Michael. Food wars: the battle for minds, mouths and markets, London: Earthscan, 2004. p. 1-10.

MALUF, Renato Sérgio; BURLANDY, Luciene; SANTARELLI, Mariana; SCHOTTZ, Vanessa; SPERANZA, Juliana Simões. Nutrition-sensitive agriculture and the promotion of food and nutrition sovereignty and security in Brazil. Ciência \& Saúde Coletiva, v. 20, n. 8, p. 2303-2312, 2015. Disponible en: <http://dx.doi.org/10.1590/1413-81232015208.14032014>. Acceso en: 1 dic. 2017.

MARQUES, Alex Cesar Cavalheiro; BARATA; Ana Júlia Senna Sarmento; ALVES; Ricardo Ribeiro. Analysis of products supply from farming family for the National School Feeding Programme (PNAE) are in the municipality of São Gabriel-RS. Ciência e Natura, v. 38, n. 2, p. 906-912, 2016. Disponible en: < http://dx.doi.org/10.5902/2179460X21889 >. Acceso en: 17 feb. 2017.

MORGAN, Kevin; SONNINO, Roberta. Sustainable development and the public realm: the power of the public plate. In: MORGAN, Kevin; SONNINO, Roberta. The school food revolution: public food and the challenge of sustainable development. London: Earthscan, 2008. p. 164-222.

MORGAN, Kevin; MORLEY, Drian. The public plate. Harnessing the power of purchase. En: MARSDEN, Terry; MORLEY, Adrian. Sustainable Food Systems Building a New Paradigm. London: Routledge, 2014. p. 84-102.

MUÑOZ, Hugo, MARTÍNEZ, Gabriel.; CARREÑO, Martha. La tributación y el gasto público agrícola en Colombia. Roma: FAO. Serie Estudios sobre Tributación Agrícola, n. 3, p. 64-67 1993.

OCAMPO, José Antonio. Reforma del estado y desarrollo económico y social en Colombia. Análisis político. Análisis Político, v. 17, p. 3-36, 1992.

OLIVEIRA, Leandro Gomes de; BATALHA, Mário Otávio; PETTAN, Kleber Batista. Comparative assessment of the food purchase program and the national school feeding program's impact in Ubá, Minas Gerais, Brazil. Ciência Rural, Santa Maria, v. 47, n. 1, p. 1-6, 2017. Disponible en: <http://dx.doi.org/10.1590/01038478cr20160395>. Acceso en: 8 dic. 2017.

PINGALLI, Prabhu. Agricultural policy and nutrition outcomes - getting beyond the preoccupation with staple grains. Food Security, v. 7, n. 3, p. 583-591, 2015. Disponible en: <http://dx.doi.org/10.1007/s12571-015-0461-x>. Acceso en: 8 feb. 2017.

PATIÑO, Carlos; CAICEDO, Elizabeth; RANGEL, Mercedes. Pobreza y desarrollo en Colombia: su impacto sobre la infancia y la mujer. Bogotá: Unicef, DNP, 1988. p. 441.

QUINTERO, Alejandro Hernán. Ciudadanía y Sistema de Protección Social en Colombia ó nuevamente ¿ciudadanía y clase social?: Análisis de las consecuencias para la ciudadanía en Colombia de la aplicación de La Ley 100 de 1993 ó Ley del Sistema de Seguridad Social Integral. Tesis (de Maestría en Ciencias Políticas, Consejo Latinoamericano de Ciencias Sociales (CLACSO), Instituto de Estudios Políticos (IEP-UDEA). Universidad de Antioquia, Medellín, Colombia.

RUSSELL, Jacqueline; DWYER, John; MACASKILL, Lesley; EVERS, Susan; UETRECHT, Connie; DOMBROW, Carol. Perceptions of Child Nutrition 
Programs: The Voices of Children, Parents, Volunteers, Program Coordinators and Educators. Journal of Hunger \& Environmental Nutrition, v. 2, n. 4, p. 47-65, 2008. Disponible en: <https://doi.org/10.1080/19320240802032453>. Acceso en: 21 feb. 2017.

SOARES, Panmela; DAVÓ-BLANES, Carmen; MARTINELLI, Suellen; MELGAREJO, Leonardo; CAVALLI, Suzi. The effect of new purchase criteria on food procurement for the Brazilian school feeding program. Appetite, v. 108, p. 288-294, 2017. Disponible en: <https://doi.org/10.1016/j.appet.2016.10.016>. Acceso en: 20 feb. 2017.

SOARES, Panmela, MARTINELLI, Suellen; MELGAREJO, Leonardo; DAVÓ-BLANES, Carmen; CAVALLI, Suzi. Strengths and weaknesses in the supply of school food resulting from the procurement of family farm produce in a municipality in Brazil. Ciência \& Saúde Coletiva, v. 20, n. 6, p. 1891-1900, 2015. Disponible en: <https://10.1590/1413-81232015206.16972014 >. Acceso en: 20 feb. 2017.

SOUZA-ESQUERDO, Vanilde, BERGAMASCO, Sonia Maria. Análise sobre o acesso aos programas de políticas públicas da agricultura familiar nos municípios do circuito das frutas (SP). Revista de Economia e Sociologia Rural, v. 52, n. 1, p. S205-S222, 2014. Disponible en: <http://dx.doi.org/10.1590/S010320032014000600011>. Acceso en: 3 mar. 2017.

SULEMANA, Mohammed; NGAH, Ibrahim; MAJID, Rafee. The challenges and prospects of the school feeding programme in Northern Ghana. Development in Practice, v. 23, n. 3, p. 422-432, 2013. Disponible en: <https://doi.org/10.1080/09 614524.2013.781127>. Acceso en: 10 feb. 2017.

UDUKU, Ola. School building design for feeding programmes and community outreach: Insights from Ghana and South Africa. International Journal of Educational Development, v. 31, n. 1, p. 59-66, 2011. Disponible en: <https:// doi.org/10.1016/j.ijedudev.2010.06.005>. Acceso en: 7 feb. 2017.

URIBE, Fani. El Instituto de Bienestar Familiar. Del asistencialismo paternalista a la participación comunitaria. Tesis de Grado - Nova University-CINDE, Medellín, Colombia, 1988.

VILLAR, Betzabeth Slater, SCHWARTZMAN, Flávia; JANUÁRIO, Bruna Lourenço; RAMOS, Jamile Fernandes. Situação dos municípios do estado de São Paulo com relação à compra direta de produtos da agricultura familiar para o Programa Nacional de Alimentação Escolar (PNAE). Revista Brasileira de Epidemiologia, v. 16, n. 1, p. 223-226, 2013. Disponible en: <http://dx.doi. org/10.1590/S1415-790X2013000100021 >. Acceso en: 17 feb. 2017.

WORLD FOOD PROGRAMME (WFP). P4P Purchase for Progress - A Primer. Roma: WFP, 2012. Disponible en: <http://documents.wfp.org/stellent/ groups/public/documents/reports/wfp245374.pdf>. Acceso en: 6 dic. 2017.

State of School Feeding Worldwide. Roma: WFP, 2013. Disponible en: $\langle$ https://www.wfp.org/content/state-school-feeding-worldwide-2013>. Acceso en: 6 dic. 2017.

Submetido em: $02 / 07 / 2018$

Aceito em: 06/11/2018 


\title{
THE CONTRIBUTION OF PUBLIC FOOD FOR RURAL DEVELOPMENT - REFLECTIONS FROM THE COLOMBIAN CASE
}

\begin{abstract}
This case study aims to analyse how the public feeding has been disputed under different conceptions. It is a field of confrontations between different actors that reconstruct rules and define discontinuous historical trajectories. Methodologically, a systematic review of secondary sources is used, as well as a primary information analysis collected by participant observation and semi-structured interviews with key actors in Colombia, mobilizing concepts from the theory of strategic action fields. Public feeding can be repositioned as a mechanism of social construction by promoting alternative agrifood networks through local purchases. However, this field has been co-opted into social and political disputes over complex dynamics that perpetuate the appropriation of agri-food systems by private companies and intermediaries. On the other hand, there is a limited capacity of the State to promote sustainable production and consumption practices, while the regulations encourage disconnected assistance models. This state lack in some cases is occupied by the collective action of some social groups with common objectives. Rethink the public feeding as a mechanism of rural development is necessary to mobilize and prioritize another meanings and values to strengthen the shared interests of well-being.
\end{abstract}

Keywords: food and nutrition security, social inclusion, local purchases.

\section{A CONTRIBUIÇÃO DA ALIMENTAÇÃO PÚBLICA PARA O DESENVOLVIMENTO RURAL - REFLEXÕES DO CASO COLOMBIANO}

\begin{abstract}
Resumo
Este estudo de caso tem como objetivo analisar como a alimentação pública tem sido disputada sob diferentes concepções, sendo um campo de confrontos entre diferentes atores que reconstroem regras e traçam trajetórias históricas descontínuas. Metodologicamente, utiliza-se uma revisão sistemática de fontes secundárias, bem como a análise de informações primárias coletadas por meio de observação participante e entrevistas semiestruturadas com atores-chave na Colômbia, mobilizando conceitos a partir da Teoria dos Campos de Ação estratégica. A alimentação pública pode ser reposicionada como um mecanismo de construção social promovendo redes agroalimentares alternativas por meio de compras locais. No entanto, este campo tem sido cooptado em disputas sociais e políticas sobre dinâmicas complexas que perpetuam a apropriação de sistemas agroalimentares por empresas privadas e intermediários. Por outro lado, existe uma capacidade limitada do Estado para promover práticas sustentáveis de produção e consumo, enquanto os regulamentos encorajam modelos assistencialistas desconectados. Essa ausência do Estado, em alguns casos, é ocupada pela ação coletiva de alguns grupos sociais com objetivos comuns. A necessidade de repensar a alimentação pública como mecanismo de desenvolvimento rural mobilizaria e priorizaria outros significados e valores para fortalecer os interesses compartilhados de bem-estar.
\end{abstract}

Palavras-chave: segurança alimentar e nutricional, inclusão social, compras locais. 